\title{
Persisting media criticism of robot-assisted laparoscopic prostatectomy
}

\author{
Charles Lee Jackson
}

Received: 4 March 2010/Accepted: 22 March 2010/Published online: 23 April 2010

(C) Springer-Verlag London Ltd 2010

\section{Dear Editor,}

Having read yet another article in the media calling into question the validity of the robotic technique for prostatectomy (New York Times, February 2, 2010), I am once again puzzled by the persistence of such criticism. I cannot recall any surgical advance enduring this kind of sustained criticism in the recent past. Clearly, a skeptical view was appropriate when this technique was first introduced. It would have been irresponsible to embrace this new technology without questioning the outcomes. However, although study after study has shown equivalent oncologic and quality of life outcomes, the criticism persists. It is criticized because it has only shown equivalency and not superiority of outcomes. The robotic technique is criticized on the basis of cost. It is dismissed as the product of clever and overreaching marketing, suggesting the public has been duped. Why does this continue 9-10 years after its introduction and following tens of thousands of operations?

Most other recent advances in surgical techniques have been adopted into practice without the burden of having to prove anything more than equivalent outcomes and safety. ESWL, laparoscopic nephrectomy, laparoscopic cholecystectomy, and laparoscopic appendectomy come to mind. Even robot-assisted laparoscopic hysterectomy is being accepted into gynecologic practice without the anguished questioning that has continued to cling to robot-assisted laparoscopic prostatectomy (RALP).
I submit that what distinguishes RALP from most previous surgical advances and other robotic applications is the steep learning curve and the unique challenges posed by the trifecta of goals. Unlike other procedures, this may not be within the grasp of all who would like to adopt it into their practice. This does not, however, reduce its value as a surgical advance. To suggest that patients prefer this technique due to clever and misleading marketing is disingenuous. Patients understand the marketing for what it is. They have generally researched their options diligently and with care. They are aware that the outcomes with open and robotic surgery are equivalent. Reassured by this, they have overwhelmingly preferred the robotic approach based upon the experience of their friends and family or friends of friends and family. They understand the value of, and desire a rapid return to, the workplace and normal productivity. We need to recognize this and be guided by their preference for a reduced burden of treatment when there are equivalent outcomes. We need to do so while emphasizing to them the importance of the experience of the surgeon, regardless of technique, as the critical factor influencing the success of their surgery. Continuing to suggest their preference is the misguided result of an advertising campaign does them a disservice and is no longer relevant or appropriate.
C. L. Jackson $(\bowtie)$

Department of Urology, Memorial Robotics and Urologic

Cancer, Memorial Health System/CHI, Suite E-790,

725 Glenwood Dr., Chattanooga, TN 37404, USA

e-mail: lee.jackson@memorial.org 